${ }^{1} \mathrm{~S}$ Willcox, ${ }^{2} \mathrm{P}$ Butt, ${ }^{3} \mathrm{~T}$ Hakaraia-Tino, ${ }^{4} \mathrm{~B}$ Sullivan, ${ }^{4} \mathrm{R}$ Hudson. ${ }^{1}$ WaterSafe Auckland Inc, New Zealand; ${ }^{2}$ Sport Waitakere, New Zealand; ${ }^{3}$ Safe Waitakere, New Zealand;

${ }^{4}$ Surf Lifesaving New Zealand

Background Youth are one of the most at-risk groups of drowning. From 2006-2010, 447 people drowned in New Zealand (NZ), of which $16 \%$ were aged $15-24$ years.

In Auckland, 104 people drowned in the same period; 23\% Pacific Island people, 13\% Maori and 6\% Asian, all high risk groups for drowning.

To address drowning within these groups, Wai Wise was developed in partnership with Sport Waitakere, Safe Waitakere, Surf Life Saving NZ and WaterSafe Auckland, funded by Sport NZ. Wai Wise specifically targets Pacific Island, Maori and Asian youth in Waitakere (West Auckland).

Aims/Objectives/Purpose

- To increase the skills, knowledge and confidence of youth for safer participation in aquatic activities

- Develop role models for promoting safety messages throughout the community.

Method Wai Wise is delivered over a 6-8 week period through both theory and practical sessions in the classroom, pool and outdoor environments.

\title{
Results and Outcomes
}

- 168 youth have completed Wai Wise

- Participants report significantly increasing their water safety knowledge and survival skills

- Participants report significantly increasing their confidence around water

- 24 young 'leaders' trained to promote safer behaviour and attitudes among their communities

- Wider outcomes include improved haoura

(health and wellbeing) of participants

Significance/Contribution This innovative approach combining practical pool and beach sessions, classroom learning and application through an aquatic activity develops critical thinking and decision making. Participants are able to assess and minimise risks of the activity or environment that could lead to injury or death and be role models to create safer communities for the future. 